EUROPEAN JOURNAL OF PURE AND APPLIED MATHEMATICS

Vol. 12, No. 2, 2019, 506-518

ISSN 1307-5543 - www.ejpam.com

Published by New York Business Global

\title{
Optimal control of a time delayed HIV-1 infection model
}

\author{
Nigar Ali ${ }^{1, *}$, Muhammad Ikhlaq Chohan ${ }^{2}$, Gul Zaman ${ }^{1}$ \\ ${ }^{1}$ Department of Mathematics, University of Malakand, Chakadara Dir(L), Khyber Pakhtunkhwa, \\ Pakistan \\ 2 Department of Business Administration and Accounting Al Buraimi University College, \\ Al-Buraimi Sultanate of Oman
}

\begin{abstract}
In this paper, an optimal control problem of HIV infection model of delay differential equations is taken into account. Two Optimal controls variables are explored. These optimal control variables represent reverse transcriptase inhibitors (RTIs) and protease inhibitors (PIs). The existence and uniqueness results for the optimal control pair are established. The optimality system is derived using Pontryagin's Maximum Principle and then solved numerically. Finally, conclusion is drawn.
\end{abstract}

2010 Mathematics Subject Classifications: 92D25, 49J15, 93D20.

Key Words and Phrases: HIV-1 model, Optimal control, Recombinant virus, Pontryagin's maximum principle, Objective functional.

\section{Introduction}

HIV stands for human immunodeficiency virus. It is the virus that can lead to acquired immunodeficiency syndrome, or AIDS, if not treated. Unlike some other viruses, the human body can't get rid of HIV completely, even with treatment. HIV attacks the body's immune system, specifically the CD4 cells (T cells), which help the immune system fight off infections. No effective cure currently exists, but with proper medical care, HIV can be controlled. But with proper medical care, HIV can be controlled.

Mathematical models have evaluated a wide range of different HIV prevention and treatment programmes. Mathematical modeling over the years has been useful in analyzing various diseases dynamics, such as HIV/AIDS, Malaria and Tuberculosis, and also plays an important role in the better understanding of epidemiological patterns for diseases control, as it provides short and long term prediction of diseases incidence. Several mathematical models have been formulated in order to understand the dynamics of HIV infection

*Corresponding author.

DOI: https://doi.org/10.29020/nybg.ejpam.v12i2.3416

Email addresses: nigaruom@gmail.com (N.Ali),chohan@buc.edu.om (M.I.Chohan), gzaman@uom.edu.pk (G.Zaman) 
[6, 9, 13-16, 19-22]. These models are utilized to explore optimal chemotherapy treatment to avoid an excessive use of drugs. Indeed, when these drugs are administered in high dose they are toxic to the human body and cause damages. Fister et al. [16] applied optimal control on HIV-1 model and discussed the percentage effect of the chemotherapy on the interaction of the CD4+ T cells with the virus. Similarly, Joshi [12] taken into account two control variables, one for raising the immune system and the other for delaying HIV-1 progression. Garira et al. [4] used two controls strategies which simulate the effect of reverse transcriptase inhibitors (RTIs) and protease inhibitors (PIs) to integrate drug efficacy. Ghanbari and Farahi [5] obtained optimal control pair for nonlinear delay HIV model with quadratic cost functional by using Fourier series approximation.

Recent research work has been focused on optimal control problem of delayed HIV model (see for example $[7,8,10,11]$ ). All these models considered the effect of control strategy on single pathogen virus. Here, we consider the delayed HIV-1 model consists of two types of viruses, that is, pathogen virus and engineered virus.

In this paper, we develop the best strategy of treatment; more exactly we seek to search a maximum count of healthy cells with a minimum dose of the administered drugs. To introduce a control to the proposed model, we analyze the interactions of healthy CD4+T cells, single infected $\mathrm{CD} 4+\mathrm{T}$ cells, double infected cells, free virus and recombinant virus: two major categories of anti-retroviral drugs to combat HIV are reverse transcriptase inhibitors (RTIs) and protease inhibitors (PIs). RTIs prevent new HIV infection by discrupting the conversion of viral RNA into DNA inside of $\mathrm{T}$ cells. PIs reduce the number of viruses particles produced by an actively-infected $\mathrm{T}$ cells. Hence, if we denote $0 \leq u_{1}(t)<1$ the RTI control variable and $0 \leq u_{2}(t)<1$ the PI control variable equations. These controls are measurable functions satisfying $0 \leq u_{j}(t)<1$ for $j=1 ; 2$. Thus, the following optimal control problem is formulated.

$$
\begin{aligned}
\frac{d x(t)}{d t} & =\Lambda-d x(t)-\left(1-u_{1}(t)\right) \beta x(t) v(t) \\
\frac{d y(t)}{d t} & =\left(1-u_{1}(t)\right) \beta e^{-a \tau} x(t-\tau) v(t-\tau)-a y(t)-\alpha w(t) y(t) \\
\frac{d z(t)}{d t} & =\alpha w(t) y(t)-b z(t) \\
\frac{d v(t)}{d t} & =k\left(1-u_{2}(t)\right) y(t)-p v(t) \\
\frac{d w(t)}{d t} & =c z(t)-q w(t)
\end{aligned}
$$

with initial conditions

$$
x(0)=x_{0}, y(0)=y_{0}, z(0)=z_{0}, v(0)=v_{0}, w(0)=w_{0} .
$$

Here, $x(t), y(t), v(t), z(t)$ and $w(t)$ denote the densities of uninfected target cells, infected cells, free virus, double infected cells and recombinant viruses at time $t$, respectively. The transitions between different states are described by the following parameters.

(i) $\Lambda$ is the recruitment rate of uninfected cells. 
(ii) $\beta$ is an effective contact rate.

(iii) $d$ is the natural mortality rate.

(iv) a stands for death rate of infected cells.

$(v) k$ is the rate of virus production.

(vi) $\alpha$ is the infection rate of pathogen infected cells.

(vii) $p$ is the death rate of pathogen virus.

(viii) $q$ is the death rate of recombinant virus.

$(i x) c$ is the rate of production of double infected cells.

(x) $\tau$ can be regarded as the average time for a viral particle to go through the eclipse phase (or average latent period).

Our target is to find the control functions $u_{1}(t)$ and $u_{2}(t)$ to get the following achievements.

(i) To maximize the density of uninfected cells and recombinant viruses during the time period $[0, T]$.

(ii) To minimize the cost of treatment, the density of pathogen virus and the density of infected cells.

In order to achieve our target, we construct the following objective functional:

$$
J\left(u_{1}(t), u_{2}(t)\right)=\int_{0}^{T}\left(\rho_{1} x(t)+\rho_{2} w(t)-\frac{1}{2}\left(\xi_{1} u_{1}^{2}(t)+\xi_{2} u_{2}^{2}(t)\right)\right) d t,
$$

where $\rho_{1}$ and $\rho_{2}$ are weight constants which balance the size of the optimal conditions and the parameters $\xi_{1} \geq 0$ and $\xi_{1} \geq 0$ are established on the benefits and costs of the treatment. These parameters equalize the size of the terms $u_{1}(t)$ and $u_{2}(t)$ which reflect the severeness of the side effects of the drugs.

To find the basic reproductive number, we follow the techniques presented in $[1-3]$ for the control problem is given by

$$
R_{0}(t)=\frac{k \beta \Lambda e^{-a \tau}\left(1-u_{1}(t)\right)\left(1-u_{2}(t)\right)}{a d p} .
$$

Clearly, the value of $R_{0}$ s decreased by increasing the values of $u_{1}(t)$ and $u_{2}(t)$. But the infection starts if $R_{0}>1$. For the system (1), with time delays $\tau=0$, the disease-free equilibrium always exists.

$$
E_{0}=\left(\frac{\Lambda}{d}, 0,0,0,0\right)
$$


If $R_{0} \leq 1$ or $\left(1-u_{1}(t)\right)\left(1-u_{2}(t)\right) \leq \frac{a p d}{k \beta \Lambda}$, then the infection would die out. If $R_{0}>1$ or $(1-$ $\left.u_{1}(t)\right)\left(1-u_{2}(t)\right) \geq \frac{a p d}{k \beta \Lambda}$, then there exists pathogen present equilibrium $E_{p}\left(x_{1}, y_{1}, z_{1}, v_{1}, w_{1}\right)$ where,

$$
\begin{aligned}
x_{1} & =\frac{a p}{\left(1-u_{1}(t)\right)\left(1-u_{2}(t)\right) \beta k e^{-a \tau}}, \\
y_{1} & =\frac{\Lambda k \beta e^{-a \tau}\left(1-u_{1}(t)\right)\left(1-u_{2}(t)\right)-a d p}{k a \beta e^{-a \tau}\left(1-u_{1}(t)\right)\left(1-u_{2}(t)\right)}, \\
z_{1} & =0, \\
v_{1} & =\frac{\Lambda k \beta e^{-a \tau}\left(1-u_{1}(t)\right)\left(1-u_{2}(t)\right)-a d p}{\left(1-u_{1}(t)\right) p a \beta e^{-a \tau}} \\
w_{1} & =0 .
\end{aligned}
$$

The recombinant present equilibrium $E_{r}\left(x_{2}, y_{2}, z_{2}, v_{2}, w_{2}\right)$ is given by

$$
\begin{aligned}
x_{2} & =\frac{(\alpha \lambda c+\gamma b q) p}{\alpha c d p+\beta k q b e^{-a \tau}\left(1-u_{1}(t)\right)\left(1-u_{2}(t)\right)}, \\
y_{2} & =\frac{b q}{\alpha c}, \\
z_{2} & =\frac{q}{\alpha c}\left(\frac{\left(1-u_{1}(t)\right)\left(1-u_{2}(t)\right) c k \Lambda \alpha \beta e^{-a(\tau)}-a \alpha c d p-\left(1-u_{1}(t)\right)\left(1-u_{2}(t)\right) a b q k \beta e^{-a \tau}}{\alpha c d p+b k q \beta e^{-a \tau}}\right), \\
v_{2} & =\frac{k q b}{\alpha c p}, \\
w_{2} & =\frac{\alpha c k \beta \lambda e^{-a(\tau)}-a \alpha c d p\left(1-u_{1}(t)\right)\left(1-u_{2}(t)\right)-\left(1-u_{1}(t)\right)\left(1-u_{2}(t)\right) a b q k \beta e^{-a \tau}}{\alpha\left(\alpha c d p+\left(1-u_{1}(t)\right)\left(1-u_{2}(t)\right) b k q \beta e^{-a \tau}\right)} .
\end{aligned}
$$

We find optimal control functions $u_{1}^{\star}(t)$ and $u_{2}^{\star}(t)$ such that

$$
J\left(u_{1}^{\star}(t), u_{2}^{\star}(t)\right)=\max \left\{J\left(u_{1}(t), u_{2}(t)\right) \backslash\left(u_{1}(t), u_{2}(t)\right) \in U\right\},
$$

where $U=\left\{\left(u_{1}(t), u_{2}(t)\right) \backslash u_{i}\right.$ is lebesgue measurable on $\left.[0,1], 0 \leq u_{i}(t) \leq 1, i=1,2\right\}$, is the control set.

\section{Existence of control problem}

In this section, we show the existence of the control problem. To do this, first we find the lagrangian of the optimal control problem.

$$
L(t)=\rho_{1} x(t)+\rho_{2} w(t)-\frac{1}{2}\left(\xi_{1} u_{1}^{2}(t)+\xi_{2} u_{2}^{2}(t)\right) .
$$

The corresponding Hamiltonian can be defined as

$$
H\left(x, y, z, v, w, x_{\tau}, v_{\tau}, u_{1}, u_{2}, \lambda(t)\right)=\frac{1}{2}\left(\xi_{1} u_{1}^{2}+\xi_{2} u_{2}^{2}\right)-\rho_{1} x(t)-\rho_{2} w(t)
$$




$$
\begin{aligned}
& +\lambda_{1}(t)\left(\Lambda-d x(t)-\left(1-u_{1}(t)\right) \beta x(t) v(t)\right) \\
& +\lambda_{2}(t)\left(\left(1-u_{1}(t)\right) \beta e^{-a \tau} x_{\tau} v_{\tau}-a y(t)-\alpha w(t) y(t)\right) \\
& +\lambda_{3}(t)(\alpha w(t) y(t)-b z(t))+\lambda_{4}(t)\left(k\left(1-u_{2}(t)\right) y(t)\right. \\
& -p v(t))+\lambda_{5}(t)(c z(t)-q w(t))
\end{aligned}
$$

where $x_{\tau}:=x(t-\tau)$ and $v_{\tau}:=v(t-\tau)$.

This Hamiltonian determines the control functions for the proposed optimal control problem.

To show the existence of optimal pair, we use the idea of Fleming and Rishel in [? ].

Theorem 3.1: For the control problem with the model (1), there always exists $u^{\star}=$ $\left(u_{1}^{\star}, u_{2}^{\star}\right) \in U$ such that

$$
\max _{\left(u_{1}(t), u_{2}(t)\right) \in U} J\left(u_{1}(t), u_{2}(t)\right)=J\left(u_{1}^{\star}(t), u_{2}^{\star}(t)\right) .
$$

Proof: To prove the existence of optimal control, we will follow [? ].

We see that the set of controls and state variables are nonnegative and nonempty [17]. The optimal system is bounded which determines the compactness needed for the existence of the optimal control. Also, the state system is bounded by a linear function in the state and control variables. Therefore, the control set $U$ is convex and closed. Using the boundedness of the solution, we see that the RHS of the integrand of the objective functional is concave on $U$. Finally, we can prove that there exist constants $h_{1}, h_{2}>0$, and $\eta>1$ such that the integrand $L\left(x(t), w(t), u_{1}(t), u_{2}(t)\right)$ of the objective functional satisfies

$$
L\left(x(t), w(t), u_{1}(t), u_{2}(t)\right)=h_{2}-h_{1}\left(\left|u_{1}\right|^{2}+\left|u_{2}\right|^{2}\right)^{\eta / 2} .
$$

Thus, conclude that there exists an optimal control.

Next, we use Pontryagin's Maximum Principle [18] to discuss the following theorem.

Theorem 3.2: For the optimal control $u_{1}^{\star}(t), u_{2}^{\star}(t)$ and solutions $x^{\star}(t), y^{\star}(t), z^{\star}(t), v^{\star}(t)$, and $w^{\star}(t)$ of the corresponding state system (1), there are adjoint variables $\lambda_{i}(t), i=$ $1,2, \ldots, 5$, satisfying the equations

$$
\begin{aligned}
\frac{d \lambda_{1}}{d t} & =\rho_{1}+\lambda_{1}(t)\left(d+\left(1-u_{1}^{\star}(t)\right) \beta v^{\star}(t)\right)+\lambda_{1}(t+\tau) \lambda_{2}(t) \beta e^{-a \tau} v^{\star}(t-\tau)\left(u_{1}^{\star}(t)-1\right), \\
\frac{d \lambda_{2}}{d t} & =a \lambda_{2}(t)+\left(\lambda_{2}(t)-\lambda_{3}(t)\right) \alpha w^{\star}(t)+\lambda_{4} k\left(u_{1}^{\star}(t)-1\right), \\
\frac{d \lambda_{3}}{d t} & =b \lambda_{3}(t)-c \lambda_{5}(t), \\
\frac{d \lambda_{4}}{d t} & =\lambda_{1}(t)\left(1-u_{1}^{\star}(t)\right) \beta x^{\star}(t-\tau)+\lambda_{4}(t+\tau) \lambda_{2}(t) \beta e^{-a \tau} x^{\star}(t)\left(u_{1}^{\star}(t)-1\right)+\lambda_{4}(t) p, \\
\frac{d \lambda_{5}}{d t} & =\rho_{2}+\left(\lambda_{2}(t)-\lambda_{3}(t)\right) \alpha y^{\star}(t)+\lambda_{5}(t) q
\end{aligned}
$$

with transversality conditions

$$
\lambda_{j}(T)=0, j=1,2, \ldots, 5 .
$$


Proof: Using Pontryagin's Minimum Principle presented in [18], we get the following system of adjoint variables

$$
\begin{aligned}
\frac{d \lambda_{1}}{d t} & =-\frac{\partial H(t)}{\partial x}-\lambda_{1}(t+\tau) \frac{\partial H}{\partial x_{\tau}}, \lambda_{1}(T)=0, \\
\frac{d \lambda_{2}}{d t} & =-\frac{\partial H(t)}{\partial y}, \lambda_{2}(T)=0, \\
\frac{d \lambda_{3}}{d t} & =-\frac{\partial H(t)}{\partial z}, \lambda_{3}(T)=0, \\
\frac{d \lambda_{4}}{d t} & =-\frac{\partial H(t)}{\partial v}(t)-\lambda_{4}(t+\tau) \frac{\partial H(t}{\partial v_{\tau}}, \lambda_{4}(T)=0, \\
\frac{d \lambda_{5}}{d t} & =-\frac{\partial H(t)}{\partial w}, \lambda_{5}(T)=0 .
\end{aligned}
$$

Further, adjusting $x(t)=x^{\star}(t), y(t)=y^{\star}(t), z(t)=z^{\star}(t), v(t)=v^{\star}(t)$ and $w(t)=w^{\star}(t)$, we get the adjoint system (5) satisfying tranversality conditions $\lambda_{j}(T)=0, j=1,2, \ldots, 5$.

Theorem 3.3: The control pair $\left(u_{1}^{\star}(t), u_{2}^{\star}(t)\right)$, which maximizes the objective functional $J$ over the region $U$ is given by

$$
\begin{aligned}
& \left.u_{1}^{\star}(t)=\max \left\{\min \left\{\frac{\beta}{\xi_{1}}\left(\lambda_{2}(t) e^{-a \tau} x^{\star}(t-\tau) v^{\star}(t-\tau)\right)-\lambda_{1}(t) x^{\star}(t) v^{\star}(t)\right), 1\right\}, 0\right\}, \\
& u_{2}^{\star}(t)=\max \left\{\min \left\{\frac{\lambda_{4}(t) k y^{\star}(t)}{\xi_{2}}, 1\right\}, 0\right\} .
\end{aligned}
$$

Proof: The optimality conditions yields the following

$$
\frac{\partial H}{\partial u_{1}}=\xi_{1} u_{1}^{\star}(t)+\lambda_{1}(t) \beta x^{\star}(t) v^{\star}(t)-\lambda_{2}(t) \beta e^{-a \tau} x^{\star}(t-\tau) v^{\star}(t-\tau),
$$

and

$$
\frac{\partial H}{\partial u_{2}}=\xi_{2} u_{2}^{\star}(t)-\lambda_{4}(t) k y^{\star}(t) .
$$

Solving equations (7) and (8) for the optimal control variables $u_{1}^{\star}(t)$ and $u_{2}^{\star}(t)$, we get

$$
\begin{gathered}
\left.u_{1}^{\star}(t)=\frac{\beta}{\xi_{1}}\left(\lambda_{2}(t) e^{-a \tau} x^{\star}(t-\tau) v^{\star}(t-\tau)\right)-\lambda_{1}(t) x^{\star}(t) v^{\star}(t)\right), \\
u_{2}^{\star}(t)=\frac{\lambda_{4}(t) k y^{\star}(t)}{\xi_{2}} .
\end{gathered}
$$

By using the property of control space, the equations (9) and (10) can be written as

$$
u_{1}^{\star}(t)=\left\{\begin{array}{l}
\left.0 \text { if } \frac{\beta}{\xi_{1}}\left(\lambda_{2}(t) e^{-a \tau} x^{\star}(t-\tau) v^{\star}(t-\tau)\right)-\lambda_{1}(t) x^{\star}(t) v^{\star}(t)\right) \leq 0, \\
\left.\frac{\beta}{\xi_{1}}\left(\lambda_{2}(t) e^{-a \tau} x^{\star}(t-\tau) v^{\star}(t-\tau)\right)-\lambda_{1}(t) x^{\star}(t) v^{\star}(t)\right) \text { if } \\
\left.0<\frac{\beta}{\xi_{1}}\left(\lambda_{2}(t) e^{-a \tau} x^{\star}(t-\tau) v^{\star}(t-\tau)\right)-\lambda_{1}(t) x^{\star}(t) v^{\star}(t)\right)<1, \\
\left.1 \text { if } \frac{\beta}{\xi_{1}}\left(\lambda_{2}(t) e^{-a \tau} x^{\star}(t-\tau) v^{\star}(t-\tau)\right)-\lambda_{1}(t) x^{\star}(t) v^{\star}(t)\right) \geq 1 .
\end{array}\right.
$$




$$
u_{2}^{\star}(t)= \begin{cases}0 \text { if } \frac{\lambda_{4}(t) k y^{\star}(t)}{\xi_{2}} & \leq 0 \\ \frac{\lambda_{4}(t) k y^{\star}}{\xi_{2}} \text { if } & 0<\frac{\lambda_{4}(t) k y^{\star}(t)}{\xi_{2}}<1, \\ 1 \text { if } \frac{\lambda_{4}(t) k y^{\star}(t)}{\xi_{2}} \geq 1 .\end{cases}
$$

The above two equations for $u_{1}^{\star}(t)$ and $u_{2}^{\star}(t)$ can be written as (using compact notation)

$$
\begin{gathered}
\left.u_{1}^{\star}(t)=\max \left\{\min \left\{\frac{\beta}{\xi_{1}}\left(\lambda_{2}(t) e^{-a \tau} x^{\star}(t-\tau) v^{\star}(t-\tau)\right)-\lambda_{1}(t) x^{\star}(t) v^{\star}(t)\right), 1\right\}, 0\right\}, \\
u_{2}^{\star}(t)=\max \left\{\min \left\{\frac{\lambda_{4}(t) k y^{\star}(t)}{\xi_{2}}, 1\right\}, 0\right\} .
\end{gathered}
$$

Here, we call formula (11) and (12) for $u_{1}^{\star}(t)$ and $u_{2}^{\star}(t)$, the characterization of the optimal control.

Therefore, we get the following optimality system.

$$
\begin{aligned}
\frac{d x^{\star}(t)}{d t} & =\Lambda-d x^{\star}(t)-\beta x^{\star}(t) v^{\star}(t)\left(1-\max \left\{\operatorname { m i n } \left\{\frac{\beta}{\xi_{1}}\left(\lambda_{2}(t) e^{-a \tau} x^{\star}(t-\tau) v^{\star}(t-\tau)\right)\right.\right.\right. \\
& \left.\left.\left.\left.-\lambda_{1}(t) x^{\star}(t) v^{\star}(t)\right), 1\right\}, 0\right\}\right), \\
\frac{d y^{\star}(t)}{d t} & =\left(1-\max \left\{\operatorname { m i n } \left\{\frac{\beta}{\xi_{1}}\left(\lambda_{2}(t) e^{-a \tau} x^{\star}(t-\tau) v^{\star}(t-\tau)\right)\right.\right.\right. \\
& \left.\left.\left.\left.-\lambda_{1}(t) x^{\star}(t) v^{\star}(t)\right), 1\right\}, 0\right\}\right) \beta x^{\star}(t-\tau) v^{\star}(t-\tau)-a y^{\star}(t)-\alpha w^{\star}(t) y^{\star}(t), \\
\frac{d z^{\star}(t)}{d t} & =\alpha y^{\star}(t) w^{\star}(t)-b z^{\star}(t), \\
\frac{d v^{\star}(t)}{d t} & =k\left(1-\max \left\{\min \left\{\frac{\lambda_{4} y^{\star}(t)}{\xi_{2}}, 1\right\}, 0\right\}\right) y^{\star}(t)-p v^{\star}(t), \\
\frac{d w^{\star}(t)}{d t} & =c z^{\star}(t)-q w^{\star}(t),
\end{aligned}
$$


along with equations (2) and (6), and Hamiltonian $H^{\star}$

at $\left(x^{\star}, y^{\star}, z^{\star}, v^{\star}, w^{\star}, x_{\tau}^{\star}, v_{\tau}^{\star}, u_{1}^{\star}, u_{2}^{\star}, \lambda_{1}, \lambda_{1}, \lambda_{2}, \lambda_{3}, \lambda_{4}, \lambda_{5},\right)$

$$
\begin{aligned}
& H^{\star}(t)=\frac{1}{2}\left(\xi_{1}\left(\max \left\{\min \left\{\frac{\beta}{\xi_{1}}\left(\lambda_{2}(t) e^{-a \tau} x^{\star}(t-\tau) v^{\star}(t-\tau)\right)-\lambda_{1}(t) x^{\star}(t) v^{\star}(t)\right), 1\right\}, 0\right\}\right)^{2} \\
& \left.+\xi_{2}\left(\max \left\{\min \left\{\frac{\lambda_{4}(t) k y^{\star}(t)}{\xi_{2}}, 1\right\}, 0\right\}\right)^{2}\right)-\rho_{1} x^{\star}(t)-\rho_{2} w^{\star}(t)+\lambda_{1}\left[\Lambda-d x^{\star}(t)\right. \\
& \left.\left.-\left(1-\max \left\{\min \left\{\frac{\beta}{\xi_{1}}\left(\lambda_{2}(t) e^{-a \tau} x^{\star}(t-\tau) v^{\star}(t-\tau)\right)-\lambda_{1}(t) x^{\star}(t) v^{\star}(t)\right), 1\right\}, 0\right\}\right) \beta x^{\star}(t) v^{\star}(t)\right] \\
& +\lambda_{2}\left[\left(1-\max \left\{\operatorname { m i n } \left\{\frac{\beta}{\xi_{1}}\left(\lambda_{2}(t) e^{-a \tau} x^{\star}(t-\tau) v^{\star}(t-\tau)\right)\right.\right.\right.\right. \\
& \left.\left.\left.\left.\left.-\lambda_{1}(t) x^{\star}(t) v^{\star}(t)\right), 1\right\}, 0\right\}\right) \beta e^{-a \tau} x^{\star}(t-\tau) v^{\star}(t-\tau)-a y^{\star}(t)-\alpha w^{\star}(t) y^{\star}(t)\right] \\
& +\lambda_{3}\left[\alpha w^{\star}(t) y^{\star}(t)-b z^{\star}(t)\right] \\
& +\lambda_{4}\left[k\left(1-\max \left\{\min \left\{\frac{\lambda_{4}(t) k y^{\star}(t)}{\xi_{2}}, 1\right\}, 0\right\}\right) y^{\star}(t)-p v^{\star}(t)\right]+\lambda_{5}\left[c z^{\star}(t)-q w^{\star}(t)\right] .
\end{aligned}
$$

To find out the optimal control and state variables, we will solve numerically the above system (13) and (14).

\section{Numerical Simulation}

In this section, we present numerical results of the optimal control problem. We solve the state system forward in time by Runge-Kutta fourth order scheme and the adjoint system by backward fourth order scheme. We illustrate a case for two different values for 50 -day treatment schedule. Some of the parameter values are considered from real data (see for more detail references [1-3]) and some are estimated. The given figures $(1-6)$ are the simulation results of our proved theoretical results.

We can make some decisions on the potency of drug therapies based on the densities of uninfected cells, infected cells, double infected cells, free virus and recombinant virus. We considered 70-day treatment and the following were observed. After some time, the number of $\mathrm{T}$ cells consistently increased throughout the treatment period, the actively infected cells and the viral load all decreased till the end of the treatment period. Fig 1 represents the concentration of $\mathrm{T}$ cells during the proposed treatment period. The $\mathrm{T}$ cell population decreases up to some time, but increases after treatment. The concentration of $\mathrm{T}$ cells increases in a logistic way. However, for a higher systemic cost, the T cell population increases at a slower rate. With both weight factors, the maximal chemotherapy is given for about the 20 days. Therefore, for the higher systemic cost, the optimal chemotherapy given produces a lower $\mathrm{T}$ cell concentration and a higher virus concentration. (Fig 2) shows that the number of infected cells approaches to a very small number after treatment, 


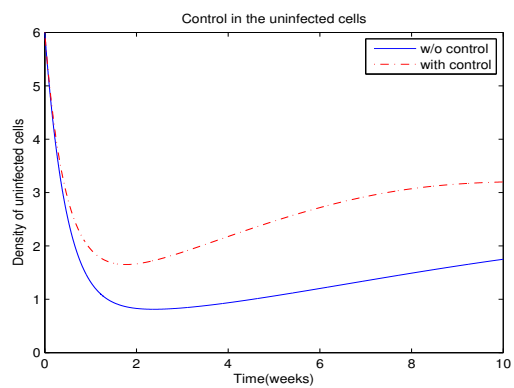

Figure 1: The graph represents in the density of uninfected cells verses time $t$ in weeks. It shows the difference in the density of uninfected cells before and after control strategy. The density of healthy cells increases after control.

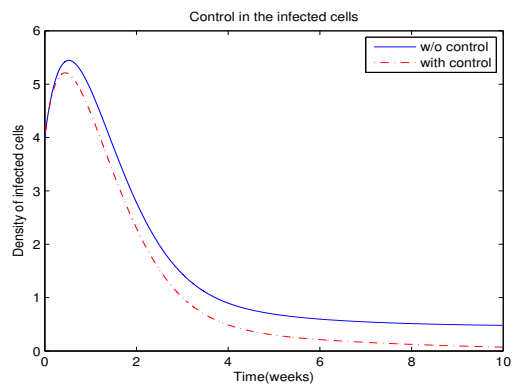

Figure 2: The graph represents control in the density of infected cells verses time $t$ in weeks. It shows the difference in the density of infected cells before and after control strategy. The density of infected cells decreases after control.

while without treatment, the number of infected cells increases. Fig 3 show that the concentration of double infected cells is reduced to a small number after treatment. Fig 4 justifies the decrease in the number of pathogen viruses after applying optimal control. The virus $\mathrm{V}$ does not cease to proliferate and so its abundance increases. After few days, the effect of chemotherapy begins to appear; which explains the growth of uninfected $\mathrm{T}$ cells and the diminishing of virus $\mathrm{V}$ (Figs. 2 and 4). Fig 5 shows that after treatment the density of recombinant virus increases with the passage of time and as a result these viruses may cause the control of infected cells. The optimal controls for drug administration are represented through Figs. 6 respectively by $u_{1}$ and $u_{2}$. 


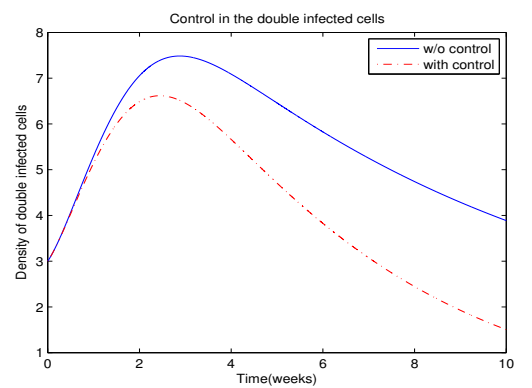

Figure 3: The graph represents control in the density of double infected cells verses time $t$ in weeks. It shows the difference in the density of double infected cells before and after control strategy. The density of double infected cells decreases after control.

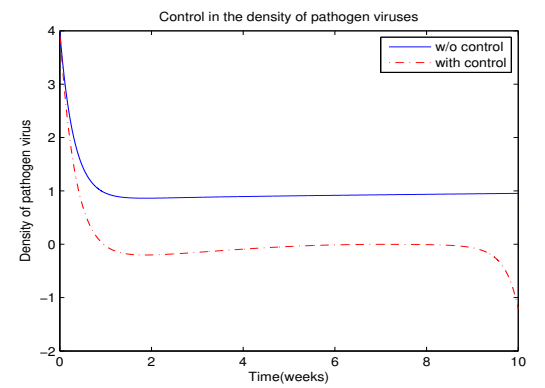

Figure 4: The graph represents control in the density of pathogen verses time $t$ in weeks. It clarify the difference in the density of pathogen virus before and after control strategy. The density of pathogen virus decreases after control decrease.

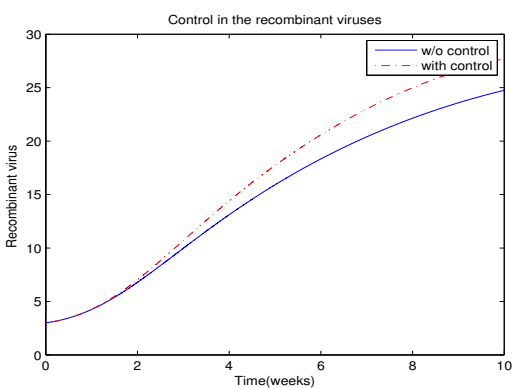

Figure 5: The graph represents control in the density of recombinant virus verses time $t$ in weeks. It shows the difference in the density of recombinant virus before and after control strategy. The density of recombinant virus increases after control. 


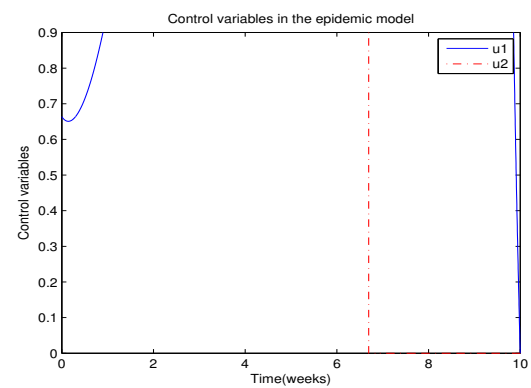

Figure 6: The graph shows the control variables in the optimal control problem.

\section{Concluding Remarks}

In this work, we have presented a single delayed HIV-1 mathematical model with two controls variables. Although, there is no effective therapy for HIV infection but different treatments are available to block off the development of the virus production in the body and maintain balance between the virus and the defense system. These treatments can cause heavy side effects such as nausea, diarrhea, fatigue, etc. Moreover, the cost of treatment is beyond reach of many infected patients. Hence, we introduced an optimal therapy in order to minimize the cost of treatment, reduce the viral load and improve the immune response. We used two controls which measure the efficacy of reverse transcriptase and protease inhibitors, respectively. In addition, we discussed an efficient numerical method based on optimal control to identify the best treatment strategy of HIV infection in order to block new infection, increase the density of recombinant viruses and minimize the number of pathogen viruses by using drug therapy with minimum side effects. The numerical simulations have shown that the viral load drops off after treatment while the densities of CD4+ cells and recombinant virus increases.

\section{Acknowledgements}

We are very thankful to the reviewers for their useful suggestions/corrections which improved this manuscript very well.

\section{References}

[1] N Ali, G Zaman. O Algahtani. Stability analysis of HIV-1 model with multiple delays. Advance Difference Equations, 1:8-19, 882016.

[2] N Ali, G Zaman. Asymptotic behavior of HIV-1 epidemic model with infinite distributed intracellular delays. SpringerPlus, 5:15-25 2016.

[3] N Ali, G Zaman, and A S Alshomrani. Optimal control strategy of HIV-1 epidemic model for recombinant virus. Cogent Mathematics, 4(1):34-45, 2017 
[4] W Garira, S Musekwa, T Shiri. Optimal control of combined therapy in a single strain HIV-1 model. Electronic Journal of Differential Equations, 5: 1-22, 2005.

[5] Gh Ghanbari and M H Farahi. Optimal control of a delayed HIV infection model via Fourier series. Journal of Nonlinear Dynamics, Article ID 495158, Volume 2014.

[6] J David, H Tran, H Banks, HIV model analysis and estimation implementation under optimal control based treatment strategies. International Journal of Pure and Applied Mathematics , 57: 357-392, 2009.

[7] Rocha D, Silva, CJ and Torres DF. Stability and optimal control of a delayed HIV model. Mathematical Methos in Applied Sciences, 9:2251-2260, 2018

[8] Wu D, Bai Y and Yu C. A new computational approach for optimal control problems with multiple time-delay. Automatica, 101: 388-395, 2019.

[9] New Hopes in HIV Disease, Science, 274: 1988-1991 (Dec.,20 1996).

[10] W H Fleming, R H Rishel. Deterministic and Stochastic Optimal Control. Springer Verlag, New York, 1975.

[11] N. Makamdop, K and Douek D C. Manipulating the Interferon Signaling Pathway. Implications for HIV Infection. Virologica Sinica, 6: 1-5, 2006

[12] H. Joshi. Optimal Control of an HIV Immunology Model. Applied Mathematics and Optimization, 23: 199- 213, 2002.

[13] D Kirschner, S Lenhart, S Serbin. Optimal Control of the Chemotherapy of HIV-1. Journal of Biological System 35: 775-792, 1997.

[14] D Kirschner, A Perelson, R Deboer. The Dynamics of HIV infectionof CD4+T Cell. Mathematical Biosciences, 114: 81-125,1993.

[15] D Kirschner, G F Webb. Immunotherapy of HIV-1 Infection, Journal of biosciences. 6(1): 71-83, 1998.

[16] K.R. Fister, S. Lenhart, J. S. Mc Nally. Optimizing Chemotherapy in an HIV Model. Electronic Journal of Differential Equations,3: 20-32, 1998.

[17] D L Lukes. Differential Equations Classical to Controlled, Mathematics in Science and Engineering. Academic Press, New York. 1982.

[18] Pontryagin L S, Boltyanskii V. G. Gamkrelidze R V, and Mishchenko E F. The Mathematical Theory of Optimal Processes, Vol 4, Gordon and Breach Science Publishers. 1986.

[19] M Shrazian and M H Farahi. Optimal Control Strategy for a Fully Determined HIV Model. Intelligent Control and Automation. 1:15-19,2010. 
[20] A Perelson, P Nelson. Mathematical models of HIV dynamics in vivo. The SIAM Review, 41:3-44, 1999.

[21] A Perelson, A Neumann, HIV-1 dynamics in vivo: Virion clearance rate, infected cell life-span, and viral generation time. Science, 271:1582-1586, 1996.

[22] X Zhou, X Song, XShi. A differential equation model of HIV infection of CD4+ Tcells with cure rate. Journal of mathematcialanaysisand applications 342: 1342-1355, 2008 . 